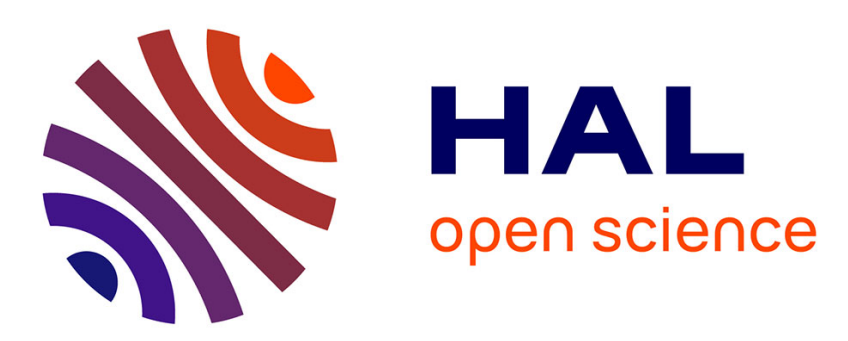

\title{
Extended Mobility Management and Routing Protocols for Internet-to-VANET Multicasting
}

Ines Ben Jemaa, Oyunchimeg Shagdar, Piedad Garrido, Francisco Martinez J, Fawzi Nashashibi

\section{- To cite this version:}

Ines Ben Jemaa, Oyunchimeg Shagdar, Piedad Garrido, Francisco Martinez J, Fawzi Nashashibi. Extended Mobility Management and Routing Protocols for Internet-to-VANET Multicasting. International Workshop on Vehicular Networking and Intelligent Transportation Systems, IEEE, Jan 2015, Las-Vegas, Nevada, United States. hal-01090204

\section{HAL Id: hal-01090204 https://hal.inria.fr/hal-01090204}

Submitted on 3 Dec 2014

HAL is a multi-disciplinary open access archive for the deposit and dissemination of scientific research documents, whether they are published or not. The documents may come from teaching and research institutions in France or abroad, or from public or private research centers.
L'archive ouverte pluridisciplinaire HAL, est destinée au dépôt et à la diffusion de documents scientifiques de niveau recherche, publiés ou non, émanant des établissements d'enseignement et de recherche français ou étrangers, des laboratoires publics ou privés. 


\title{
Extended Mobility Management and Routing Protocols for Internet-to-VANET Multicasting
}

\author{
Ines Ben Jemaa*, Oyunchimeg Shagdar*, Francisco J. Martinez ${ }^{\dagger}$, Piedad Garrido ${ }^{\dagger}$, and Fawzi Nashashibi* \\ * RITS team, INRIA Rocquencourt \\ Domaine de Voluceau, B.P. 105 78153, Le Chesnay, FRANCE \\ Email: \{ines.benjemaa, oyunchimeg.shagdar, fawzi.nashashibi\}@inria.fr \\ ${ }^{\dagger}$ Computer Science and System Engineering Department, University of Zaragoza \\ Ciudad Escolar s/n, 44003, Teruel, SPAIN \\ Email: \{f.martinez, piedad $\} @$ unizar.es
}

\begin{abstract}
Emerging ITS applications such as fleet management and point of interest distribution require vehicles to have Internet access. However, allowing vehicles to access to the Internet is particularly challenging due to the special characteristics of the vehicular environment. So far, multicasting approaches have been demonstrated to be effective for supporting group communication in traditional networks. However, such Internet-to-VANET multicast service involves several challenges including efficient multicast mobility management and multicast message delivery. This paper proposes a scheme that combines the existing multicast mobility management scheme with vehicular networking solutions to achieve Internet-to-VANET multicasting. The proposed scheme aims to: (i) provide multicast mobility management with low control overhead and efficient bandwidth utilization, as well as (ii) extend the service coverage provided by VANET membership management and multicast message delivery protocol. Simulation results indicate that our MotionMAODV scheme improves the performance of both MAODV and traditional flooding dissemination schemes in terms of both packet delivery ratio and end-to-end transmission latency.

Index Terms-Multicast, VANET, fleet management, Point of Interest, Intelligent Transportation Systems
\end{abstract}

\section{INTRODUCTION}

Vehicular ad hoc networks (VANETs) are wireless communication networks that do not require any fixed infrastructures. Vehicles function as communication nodes and relays, forming dynamic networks with other near-by vehicles on the road and highways [1], [2]. Unlike other kind of mobile networks, VANETs are characterized by: (i) constrained but highly variable network topology, (ii) specific speed patterns, (iii) time and space varying communication conditions (e.g., signal transmissions can be blocked by buildings), and (iv) no significant power constraints.

Multicasting (i.e., one-to-many or many-to-many distribution) allows communications where information is addressed to a group of destination nodes simultaneously. A large number of applications, including TV broadcasting, video conferencing, and gaming, require multicasting. With the advancement of wireless communications technologies, users can now have multicast services while they are driving. In addition to the traditional multicast applications, vehicular communications allow emerging new multicast applications such as fleet management and point of interest (POI) distribution.
Fleet management such as route guidance service to a fleet of vehicles often requires a control/service center in the Internet to provide information to a set of vehicles. As for POI distribution, it refers to informing drivers and passengers about specific location points (e.g., parking lots, restaurants, and so on), which can be interesting or useful for the nearby road users.

Multicasting in the Internet has a long history producing a number of standardized protocols to support multicast services for addressing [3], membership management, [4], and multicast routing [5], [6]. However, the specific characteristics of the vehicular environment make it difficult to extrapolate all these protocols to VANETs. In particular, multicasting to VANET mobile users (i.e., drivers and occupants) requires additional challenges including: (i) multicast mobility management, and (ii) multicast message dissemination in the wireless network.

Regarding mobility management, whose objective is to locate mobile users and provide them data in a seamless manner, two types of mobility management protocols have been standardized: (i) the Mobile IP (MIPv6 for IPv6) [7], a host-based mobility management, and (ii) the proxy mobile IP (PMIPv6) [8], which is a network-based mobility management solution. The key idea of MIPv6 relies on a fixed entity in the Mobile Node's (MN) home network, the so-called Home Agent (HA), which locates the MNs and builds a bidirectional tunnel that is used for transferring data destined to each MN. The working mode of PMIPv6 is similar, except that the visiting network localizes the $\mathrm{MN}$, communicates with the home entity, and builds a tunnel for data transfer.

In the context of multicast message dissemination, we also find standardized solutions, in which some membership management approaches are added to the MIPv6/PMIPv6 architectures, enabling the home network to forward multicast data to the MNs by using the tunnel [4].

Although the existing multicast mobility management solutions can provide multicast data to mobile nodes (MNs), an issue of the solutions is that they are somehow based on the assumption that users usually stay in their home network (fixed network), while considering the mobility of only one or few users. Therefore, the simple and direct application of 
these solutions to the emerging vehicular services, where there are many vehicles and the majority of them are continuously moving, would imply large control overhead due to per-user membership management, and inefficient bandwidth utilization due to the unicast transmissions over the tunnels. We consider that such control overhead and bandwidth utilization are not necessary, especially for the cases where the mobile users are in the same geographical area, which is the case for the abovementioned fleet management and POI distribution applications. Motivated by this, in this paper we propose an extended multicast mobility management scheme, specially designed to the vehicular communications, that provides mobility management to a set of mobile nodes with small control overhead and efficient bandwidth utilization.

In vehicular environments, another issue of the existing multicast mobility management approaches is obviously that they cannot provide multicast data to MNs that do not have Internet connection (e.g., because they are not in the coverage of access networks, and/or they are not equipped with $3 \mathrm{G} / 4 \mathrm{G}$ devices). A solution to provide multicast data to such $\mathrm{MNs}$ is to extend the mobility management solution by providing ad-hoc networking, (i.e., by using VANETs). A number of efforts towards enabling multicasting in ad-hoc networks have been previously made, especially for message dissemination [9], [10], [11].

The previously proposed message dissemination protocols for multicasting in ad-hoc networks can be divided into broadcast protocols, where the data is disseminated in the entire network, and multicast protocols, where data is sent to only a subset of nodes (i.e., the group members). In general, multicast protocols are known to perform significantly better in terms of forwarding efficiency and bandwidth utilization, because they are based on creation and maintenance of routing structures (tree or mesh). However, it is not clear that they perform better than broadcast approaches in highly mobile scenarios such as those commonly found on the vehicular environments. An earlier work [12] compared the performance of the two types of schemes for MANETs, and concluded that multicast schemes perform better than broadcast approaches when the group size (i.e., the number of multicast members with regard to the total number of nodes) is small, while it is better to use a broadcast protocol in highly mobile scenarios and/or when the group size is large. Indeed, in the research community, there is a tendency to prefer broadcast approaches (i.e., flooding, opportunistic forwarding, etc.) rather than multicast protocols.

However, the abovementioned study was made for MANETs and targeting the random way-point mobility model, which does not represent at all the specific mobility characteristics found in VANETs. Furthermore, in applications such as fleet management or POI distribution, the multicast receivers tend to move together (especially true for fleet management) and/or stay around some geographical area with low velocity (especially true for POI distribution). Considering these, in this paper we revisit a traditional multicast routing protocol and propose necessary extensions specially designed for the VANET characteristics. Specifically, we consider the application of the Multicast ad hoc on-demand distance vector (MAODV) protocol [13] for VANET multicasting, and propose an extended MAODV, we call Motion-MAODV, which has additional functionalities to handle mobility dynamics of VANETs.

The contributions of this work are the following:

- The proposed mobility management scheme aims to reduce control overhead and improve bandwidth utilization for Internet-to-VANET multicasting. This scheme is based on MIPv6 and PMIPv6 architectures, and hence, it does not require any additional infrastructure.

- To extend the coverage of the multicast service, we propose the Motion-MAODV, a VANET membership management scheme which extends the MAODV protocol. The Motion-MAODV is developed based on the link-stability study in VANET systems targeting realistic vehicular mobility.

- We compare the performance of our Motion-MAODV against both the MAODV and the traditional flooding.

The rest of this paper is organized as follows: Section II details our proposal for Internet-to-VANET multicasting. More specifically, we present the VANET multicast group management scheme and the data dissemination process to deliver information from the leader to the VANET group members. In Section III we assess our proposal by simulating different highway scenarios. Finally, Section IV concludes this paper.

\section{INTERNET-TO-VANET MULTICASTING}

In this section, we first introduce the enhanced mobility management scheme for both MIPv6 and PMIPv6 architectures, and then present our Internet-to-VANET multicasting message delivery approach.

\section{A. Extended multicast mobility management for the MIPv6 and PMIPv6 architectures}

The membership management and data transmission in the MIPv6 and PMIPv6 multicast mobility management schemes are illustrated in Figure 1. More specifically, in MIPv6, the Home Agent (HA) transmits a multicast listener query (MLQ) to the Mobile Node (MN) over the tunnel, and the MN returns a Multicast Listener Report (MLR) showing its interest to receive the multicast data. As for PMIPv6, the Mobile Access Gateways (MAGs) broadcast MLQ to MNs under their coverage, collect MLRs from them, and send aggregated MLRs to their respective Local Mobility Anchor (LMA).

Upon reception of MLR, the HA/LMA joins the multicast delivery tree and forwards received multicast data over the bidirectional tunnel(s) to the MNs/MAG for MIPv6/PMIPv6. In PMIPv6, the MAG multicasts received data to the MNs. As shown in Figure 1, the efficiency in terms of control overhead and bandwidth utilization of the two schemes (i.e., MIPv6 and PMIPv6) degrades with the increase of the number of mobile nodes because MIPv6 (resp. PMIPv6) sends data over as many unicast tunnels as the number of MNs (resp. LMAs). Moreover, the two approaches cannot obviously deliver data 


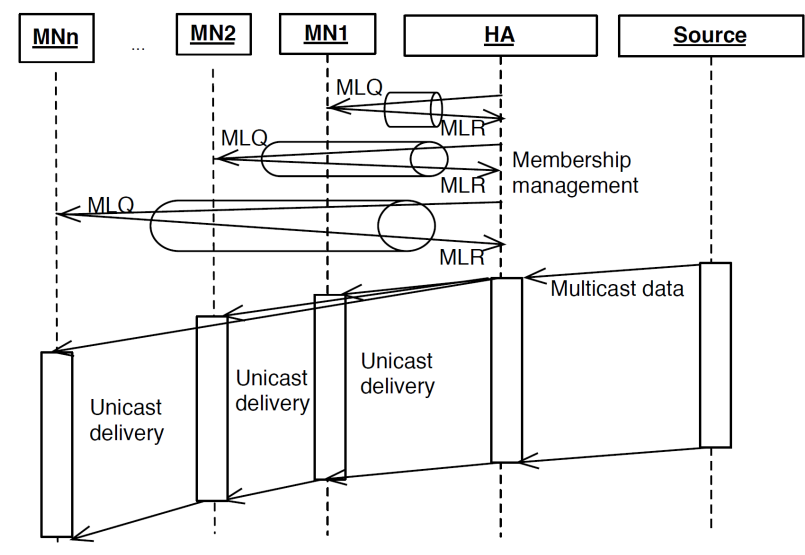

(a)

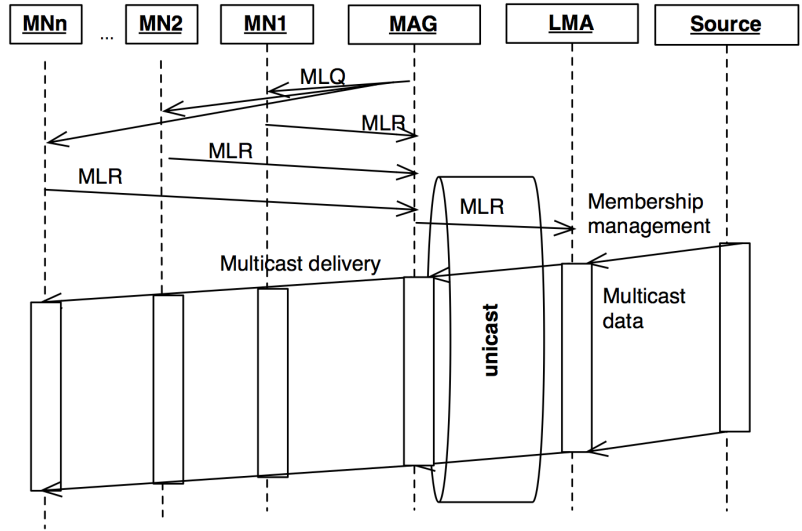

(b)

Fig. 1. Multicast mobility management solutions based on: (a) MIPv6 and (b) PMIPv6.

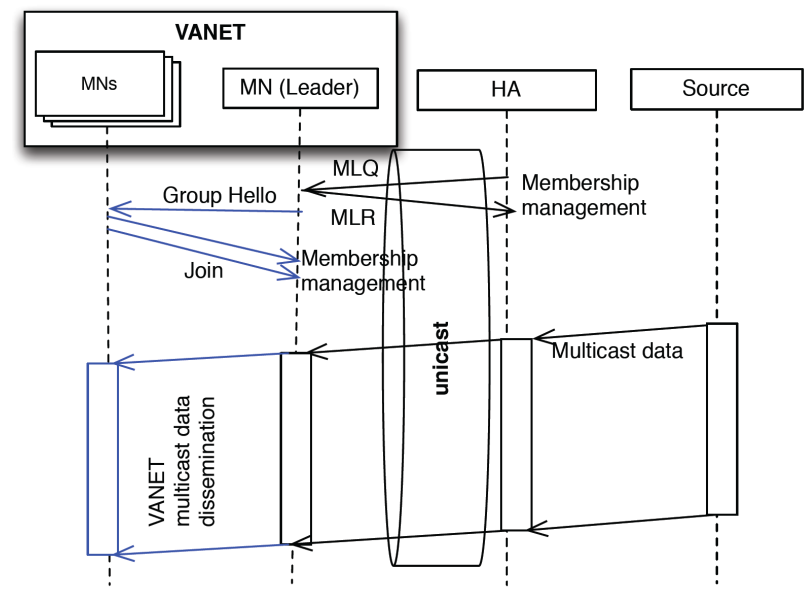

Fig. 2. Extended multicast mobility management for MIPv6.

to the MNs that do not have Internet connection either due to the signal coverage or not being equipped with the necessary communications equipment (e.g., 3G/4G devices).

Targeting the abovementioned issues, we propose to extend the multicast mobility management solutions by exploiting the VANET concept. Figure 2 illustrates the proposed scheme for MIPv6. In the proposed scheme, one MN responds to its HA's MLQ and receives multicast data from the Internet (i.e., from the HA). On parallel, the MN creates a multicast group in the VANET (i.e., it becomes the leader) to distribute multicast data to the MNs that are interested in the service. In order to prevent creating too large VANET groups, the size of the multicast group in terms of the maximum number of hops from the leader to any member would not exceed $T T L_{\max }$ number of hops. The detailed membership management and data dissemination in the VANET is described in the next subsection. Finally, it should be mentioned that the above extension can also be made for PMIPv6; the difference is that the leader would interact with a MAG instead of a HA.

\section{B. Message dissemination in VANET}

In the previous subsection we proposed an extended multicast mobility management scheme that enables a $\mathrm{MN}$ to join a multicast group in the Internet, and thereby making it possible to create VANET multicast groups in order to disseminate multicast data to nodes using vehicular networking solutions. Message dissemination from the Internet to the leader can be achieved following an Internet multicast routing protocol (e.g., Protocol Independent Multicast, PIM), and the handover functionalities of MIPv6/PMIPv6 should also be applied to support handover of the leader. Note that handover in access networks with small cell sizes (such as WLANs) is an extremely challenging task. However, in this paper we assume the use of an access technology with relatively large cell coverage (e.g., 3/4G macro cells), consequently handover is not within the scope of this paper.

The interest of this subsection is the VANET multicast group management and data dissemination from the leader to the VANET group members. As mentioned in the previous section, while there is a tendency to use broadcast schemes for multicast applications in mobile ad-hoc networks, the mobility models used are far from being realistic in VANET scenarios, and they often do not have particular applications in mind. In this paper, considering applications such as fleet management and/or POI distribution (where the mobile users often move together and/or stay in the same geographical region), we revisit a multicast protocol and study its applicability to VANET. Specifically, we study MAODV [13], which is a typical tree-based multicast routing protocol, and point out two issues that degrade its performance in vehicular scenarios. We then propose an enhanced MAODV, called Motion-MAODV that has extended functionalities to overcome the problems detected.

According to MAODV, all the VANET nodes should maintain a multicast routing table for each multicast group with the entries of the leader identity, the sequence number (which indicates the freshness of the information), and the routing cost, which is the number of hops to the leader from the node. 


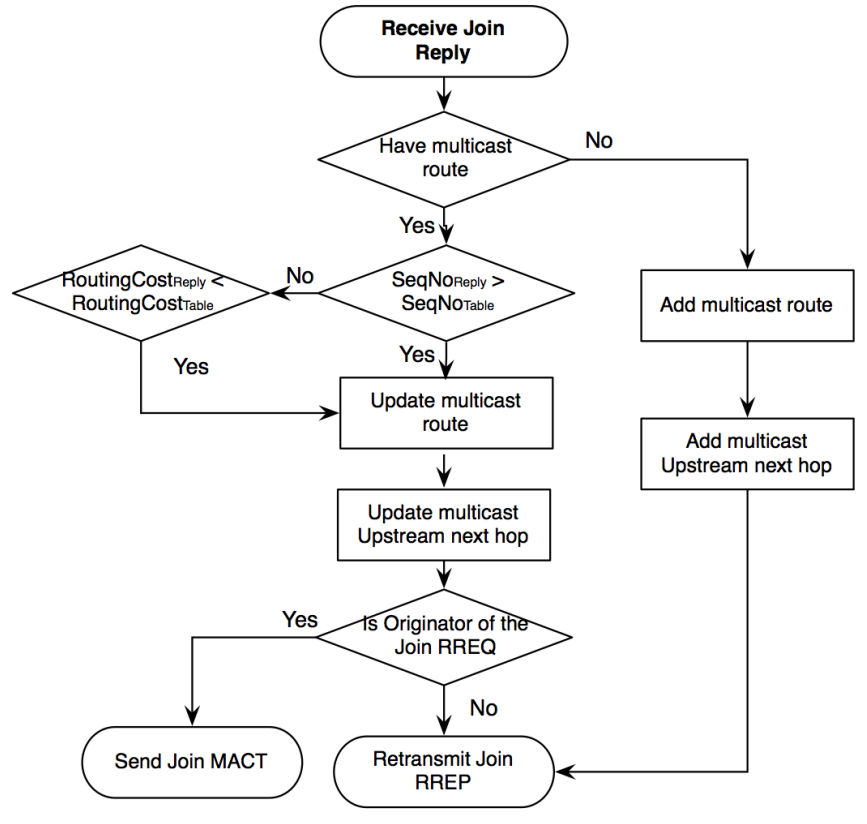

Fig. 3. Join RREP Reception process in MAODV.

The leader of the multicast group (in our case it is the node which joined the multicast group in the Internet) periodically broadcasts Group Hello messages (GRPH) to announce the existence of the group.

Figure 3 details the MAODV membership management and route configuration procedures. If a node, say node A, wishes to join a multicast group, it sends a Join Route Request (RREQ), which will be flooded in the network. A node, say node B (it can be leader), which receives the Join RREQ, responds with a Join Route Reply (RREP), if it already has a route to the leader (i.e., node $\mathrm{B}$ has an entry in the routing table). The Join RREP is transmitted following the reverse path of the RREQ. A node on the reverse path, say node C, receives the Join RREP, updates its multicast routing table with the information contained in the RREP, and retransmits the RREP. If it is not the first time to receive a Join RREP, i.e., node $\mathrm{C}$ already has an entry on the routing table, it compares the information contained in the RREP to that of the table. Node $\mathrm{C}$ updates the routing table if the sequence number of the RREP is larger than that in the table, or the sequence numbers are equal but the number of hops of the RREP is smaller than that in the table. Once the node updates the table, it retransmits the RREP. When node A receives a Join RREP, it sends a Multicast Activation Message (MACT) to node B in order to activate the route to the multicast tree.

One of the problems we see in MAODV is that it does not consider mobility dynamics of the network. Additionally, in a previous study [14], we showed that link stability, in terms of the lifetime of a link, sharply degrades with the increase of the relative velocity of the nodes: $\Delta t=R /\left|V_{e}-V_{n}\right|$, where $\mathrm{R}$ is the transmission range, whereas $V_{e}$ and $V_{n}$ are the velocity vectors of the ego vehicle and its neighbor, respectively. Our study showed that it is sufficient to express link stability with only the relative velocity between the nodes because the relative velocity has more impact on link stability than other features, such as the moving directions and the density. Considering this, our proposed Motion-MAODV works as follows:

- Each node periodically broadcasts hello messages, which contain the velocity and the positions of the neighboring vehicles, allowing each vehicle to estimate the stability of each link.

- We define a new metric called Route Stability $\left(R S_{i}\right.$ between two nodes $i$ and $j$, that calculates the cost of the route as follows:

$$
R S_{i}= \begin{cases}0 & \text { if leader } \\ \frac{\Delta V_{i j}+\left(n_{\text {hops }}-1\right) * R S_{j}}{n_{\text {hops }}} & \text { else }\end{cases}
$$

Here, $\Delta V_{i j}$ is the relative velocity of node $i$ and node $j$, $n_{\text {hops }}$ is the number of hops between node $i$ to the leader, and $R S_{j}$ is the Route Stability between node $j$ and the leader.

- Join RREP as well as the multicast routing table include $R S_{i}$.

- Upon reception of RREPs, the node first selects $n$ routes with smaller $R S$ values, and then it selects the one that has the least number of hops.

\section{PERFormance Evaluation}

In our simulations, the SUMO traffic simulator [15] is used to generate realistic vehicular mobility traces. More specifically, we simulated a highway scenario illustrated by Figure 4 . The overall length of the road is of 2 kilometers. The road has multiple forward and backward lanes. The maximum velocity of vehicles is limited to $50 \mathrm{~km} / \mathrm{h}$. The acceleration and deceleration values of the vehicles are set to $0.8 \mathrm{~m} / \mathrm{s}^{2}$ and $4.5 \mathrm{~m} / s^{2}$, respectively, and the minimum inter-vehicle distance is of 2.5 meters. Vehicles are generated at the edge of each lane following the Poisson process at the average rate $\lambda$ (in terms of vehicles/second) [16]. For each generation rate, we perform 10 simulation runs, and each simulation run lasts for 300 seconds.

More specifically, the average generation rate $\lambda$ is varied along the simulations. Additionally, the simulations are carried out for different numbers of backward and forward lanes (i.e, one, two, and three lanes per direction). Having the fleet management application in mind, the multicast members including the leader node reside on the forward lane, and the members join the multicast group at random points of time before the multicast data transmission starts. Regarding vehicle-to-vehicle (V2V) communications, we follow the IEEE $802.11 \mathrm{p}$ standard [17], and the maximum communication range considered was 300 meters. Table I summarizes the settings of our simulations.

In order to correctly assess our approach, we added to the ns-3 [18] simulator both our Motion-MAODV, and the MAODV protocol, following the specification of the IETF 


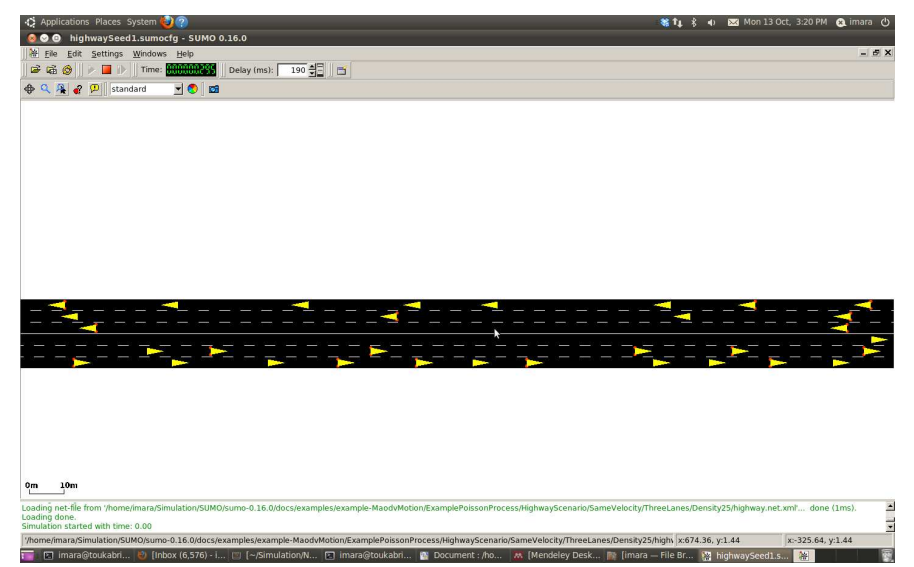

Fig. 4. Simulation scenario.

TABLE I

Simulation SETTINGS

\begin{tabular}{|l|c|}
\hline Parameter & Value \\
\hline Simulated scenario & Highway \\
\hline Simulation time & $100 \mathrm{~s}$ \\
\hline Road length & $2000 \mathrm{~m}$ \\
\hline Number of lanes & 2,4, and 6 \\
\hline Number of nodes per km & $10-95$ \\
\hline Maximum velocity of vehicles & $50 \mathrm{~km} / \mathrm{h}$ \\
\hline Acceleration of vehicles & $0.8 \mathrm{~m} / \mathrm{s}^{2}$ \\
\hline Deceleration of vehicles & $4.5 \mathrm{~m} / \mathrm{s}^{2}$ \\
\hline Minimum inter-vehicle distance & $2.5 \mathrm{~m}$ \\
\hline Maximum transmission range & $300 \mathrm{~m}$ \\
\hline Propagation model & Log-distance \\
\hline Channel bandwidth & $6 \mathrm{Mbps}$ \\
\hline
\end{tabular}

[13]. Then, we evaluated the performance of the three protocols: (i) our proposed Motion-MAODV, (ii) the MAODV, and (iii) the traditional flooding approach. In particular, we compared them in terms of packet delivery ratio (PDR), and end-to-end transmission latency.

Figure 5 shows the PDR of the three protocols when varying the vehicle density (in terms of average inter-vehicle distance per lane). As shown, when the vehicle density decreases (i.e., the inter-vehicle distance per lane increases), the PDR obtained by the three protocols also decreases. This is due to the fact that higher densities ensure the connectivity of the network. Nevertheless, flooding results in lower PDR than the tree-based approaches (i.e., MAODV and Motion-MAODV), since structureless approaches (such as flooding) can reduce their performance due to serious redundancy, contention, and massive packet collisions caused by simultaneous forwarding, especially in higher vehicle densities. In contrast, in the treebased approaches the packets are delivered only over the multicast tree, thereby reducing the redundant retransmissions. As for lower vehicle densities (i.e., higher inter-vehicle distances), our Motion-MAODV performs better than MAODV. It should be mentioned that a higher inter-vehicle distance implies an increase of the mobility dynamics, since vehicles' movements are not so affected by the rest of vehicles.

As explained in the previous section, the mobility is considered in our Motion-MAODV in such a way that the

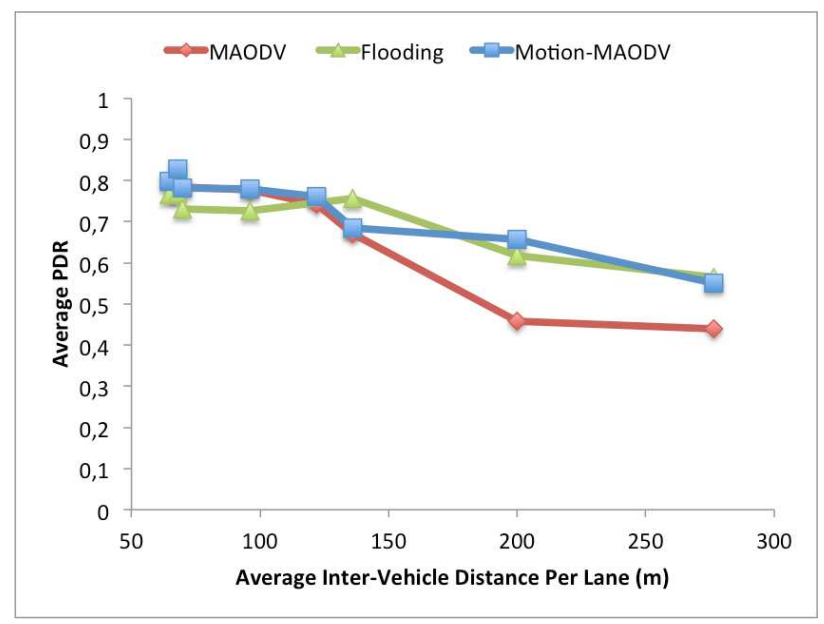

Fig. 5. Average Packet Delivery Ratio.

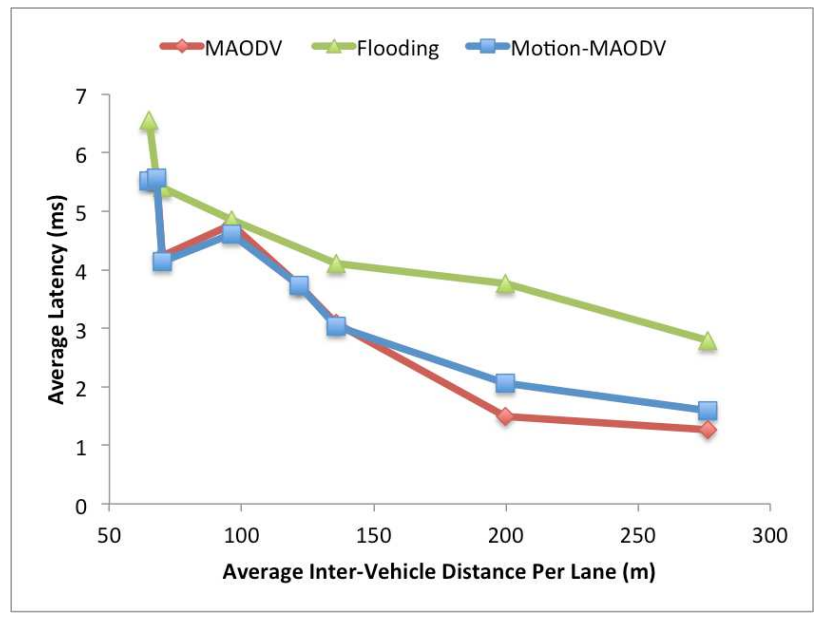

Fig. 6. Average Latency.

multicast tree is built based on Route Stability metric which accounts for the relative velocity of vehicles. This allows the multicast delivery tree of Motion-MAODV to better adapt to the mobility dynamics. On the other hand, MAODV only considers the number of hops while building the multicast tree. Consequently, in the MAODV delivery tree, the links are often broken (especially in lower vehicle densities), which results in packet loss. However, in dense scenarios, where the vehicles' velocity is low, the impact of route stability metric is small.

Figure 6 compares the average latency of the three protocols. As expected, when the vehicle density decreases, the latency also decreases for all the three protocols. However, it is clear that regardless of the vehicle density, the tree-based approaches result in shorter end-to-end delays thanks to their efficient message delivery.

Overall, we can conclude that our Motion-MAODV outperforms both MAODV and flooding, especially in lower vehicle densities, since it provides a higher PDR than MAODV while obtaining a similar latency, and a similar PDR than flooding while reducing the average latency. 


\section{CONCLUSION}

In this paper, we propose the Motion-MAODV, an extended membership management and mobility-aware tree-based multicast message dissemination protocol, specially designed to provide Internet-to-VANET multicasting with reduced control overhead and expanded service coverage. Our mobility management scheme extends the existing MIPv6 and PMIPv6 solutions by exploiting the VANET concept offering low overhead and efficient bandwidth utilization.

The performance of Motion-MAODV is assessed by using the SUMO traffic simulator and ns-3 network simulator. In particular, we compared our approach against traditional flooding and the MAODV. Simulation results show that MotionMAODV improves overall performance compared to flooding and MAODV, in terms of PDR and end-to-end latency, especially in low vehicle densities. As future work, we plan to investigate the performance of Motion-MAODV in complex urban scenarios.

\section{ACKNOWLEDGMENTS}

This work was partially supported by the Government of Aragón and the European Social Fund (T91 Research Group), as well as by the Ministry of Education of Spain within the Campus of International Excellence Programme. The authors would like to thank Pierre Merdrignac who helped in the implementation and the testing of MAODV on ns-3.

\section{REFERENCES}

[1] M. Fogue, P. Garrido, F. J. Martinez, J.-C. Cano, C. T. Calafate, and P. Manzoni, "An adaptive system based on roadmap profiling to enhance warning message dissemination in VANETs," IEEE/ACM Transactions on Networking, vol. 21, no. 3, pp. 883-895, 2013.

[2] J. A. Sanguesa, M. Fogue, P. Garrido, F. J. Martinez, J.-C. Cano, C. T. Calafate, and P. Manzoni, "An infrastructureless approach to estimate vehicular density in urban environments," Sensors, vol. 13, no. 2, pp. 2399-2418, 2013

[3] H. Holbrook, B. Cain, and B. Haberman, "Using Internet Group Management Protocol Version 3 (IGMPv3) and Multicast Listener Discovery Protocol Version 2 (MLDv2) for Source-Specific Multicast," RFC Editor, IETF, RFC 4604, August 2006. [Online]. Available: https://tools.ietf.org/html//rfc4604

[4] B. Cain, S. Deering, I. Kouvelas, B. Fenner, and A. Thyagarajan, "Internet Group Management Protocol, Version 3," RFC Editor, IETF, RFC 3376, October 2002. [Online]. Available: https://tools.ietf.org/html/rfc3376
[5] T. Pusateri, "Distance vector multicast routing protocol," RFC Editor, IETF, Internet-Draft, October 2003. [Online]. Available: http://gamay.levkowetz.com/pdf/draft-ietf-idmr-dvmrp-v3-11.pdf

[6] B. Fenner, M. Handley, H. Holbrook, and I. Kouvelas, "Protocol Independent Multicast - Sparse Mode (PIM-SM): Protocol Specification (Revised)," RFC Editor, IETF, RFC 4601, August 2006. [Online]. Available: http://tools.ietf.org/html/rfc4601

[7] D. Johnson, C. Perkins, and J. Arkko, "Mobility support in IPv6," RFC Editor, IETF, RFC 3775, June 2004. [Online]. Available: http://www.ietf.org/rfc/rfc3775.txt

[8] S. Gundavelli, K. Leung, V. Devarapalli, K. Chowdhury, and B. Patil, "Proxy mobile IPv6," RFC Editor, IETF, RFC 5213, August 2008. [Online]. Available: https://tools.ietf.org/html/rfc5213

[9] T. Le and Y. Liu, "Opportunistic Overlay Multicast in Wireless Networks," IEEE Global Telecommunications Conference (GLOBECOM), pp. 1-5, Dec. 2010.

[10] A. Sebastian, M. Tang, Y. Feng, and M. Looi, "A Multicast Routing Scheme for Efficient Safety Message Dissemination in VANET," IEEE Wireless Communication and Networking Conference (WCNC), pp. 1-6, Apr. 2010.

[11] Y.-L. Hsieh and K. Wang, "Dynamic overlay multicast for live multimedia streaming in urban VANETs," Computer Networks, vol. 56, no. 16, pp. 3609-3628, Nov. 2012.

[12] L. K. Law, S. V. Krishnamurthy, and M. Faloutsos, "Understanding and exploiting the trade-offs between broadcasting and multicasting in mobile ad hoc networks," IEEE Transactions on Mobile Computing, vol. 6, no. 3, pp. 264-279, 2007.

[13] E. Royer and C. Perkins, "Multicast ad hoc on-demand distance vector (MAODV) routing," RFC Editor, IETF, RFC, July 2000. [Online]. Available: https://tools.ietf.org/id/draft-ietf-manet-maodv-00.txt

[14] I. Ben Jemaa, O. Shagdar, P. Muhlethaler, and A. De La Fortelle, "Analysing impact of mobility dynamics on multicast routing in vehicular networks," in The Fifth International Conference on Emerging Network Intelligence (EMERGING), 2013, pp. 73-76.

[15] D. Krajzewicz, J. Erdmann, M. Behrisch, and L. Bieker, "Recent development and applications of SUMO - Simulation of Urban MObility," International Journal On Advances in Systems and Measurements, vol. 5, no. 3\&4, pp. 128-138, December 2012.

[16] S. Busanelli, G. Ferrari, and R. Gruppini, "Performance analysis of broadcast protocols in VANETs with Poisson vehicle distribution," in 11th International Conference on ITS Telecommunications (ITST), August 2011, pp. 133-138.

[17] IEEE 802.11 Working Group, "IEEE Standard for Information Technology - Telecommunications and information exchange between systems - Local and metropolitan area networks -Specific requirements Part 11: Wireless LAN Medium Access Control (MAC) and Physical Layer (PHY) specifications Amendment 6: Wireless Access in Vehicular Environments," July 2010.

[18] "Network Simulator NS-3," 2014. [Online]. Available: http://www.nsnam.org/ 